\subsection{1}

doi: 10.15789/2220-7619-2018-4-6.61

MOLECULAR EPIDEMIOLOGY OF TUBERCULOSIS IN MONGOLIA: SOURCES AND PATHWAYS OF MDR MYCOBACTERIUM TUBERCULOSIS STRAINS

S.N. Zhdanova ${ }^{1}$, T. Oyuntuya ${ }^{2}$, M.V. Badleeva ${ }^{3}$, O.B. Ogarkov ${ }^{1,4}$

${ }^{I}$ Scientific Center of Family Health and Human Reproduction, Irkutsk, Russia; ${ }^{2}$ National Center for Infectious Diseases, Ulaanbaatar, Mongolia: ${ }^{3}$ Buryat State University, Ulan-Ude, Russia $:{ }^{4}$ Branch of the Educational Institution of Further Professional Education «Russian Medical Academy of Continuing Professional Education», Irkutsk, Russia

Mongolia is a country with a high burden of tuberculosis (TB). The emergence and spread of multidrug resistance (MDR) TB in Mongolia is associated with problems of early diagnosis and the possibility of cross-border spread of MDR-TB along the Trans-Siberian Railway line from Russia or China. The objective of this study was to reveal sources and pathways of Mycobacterium tuberculosis (MTB) strains in Mongolia.

A total of DNAs of MTB from Mongolia (309 strains) were studied. RD 105/207 and 24 loci MIRU-VNTR typing were applied for genotyping and the results were analyzed by MIRU-VNTRplus application. PCR-real time typing was used to identify subtype CC2/W148 by the specific deletion in the $k d p D$ gene. Mongolian MIRU-VNTR patterns of MTB were compared with evaluable published Chinese and Russian profiles.

All tested 309 MTB isolates distributed to four lineages: Beijing (228/309 - 73.8\%), LAM (33/309 - 10.7\%), T $(30 / 309-9.7 \%), \mathrm{H}(9 / 309-2.9 \%)$, and orphan $(9 / 309-$ 2.9\%). 21 clusters uniting 187 strains were identified. Out of 228 Beijing strains 165 were clustered and significantly associated with MDR-TB cases from Ulaanbaatar and big railway stations settlements $(\mathrm{p}<0.001)$, but MIRU-VNTR profiles from all Mongolian provinces were common.

24-MIRU-VNTR profiles of Mongolian and Russian strains were differed. The three largest clusters consisted of Beijing strains with MLVA MtbC15-9 profiles of 342-32 (58 isolates), 3819-32 (33 isolates) and 1773-32 (33 isolates). Strains with such profiles were found among Russian isolates in single cases in the Irkutsk, Buryat and Zabaikal regions. Subtype CC2/W148, which was primary source of MDR strains in Russia, not found among Beijing strains in Mongolian cohort. Phylogenetic analysis established high genetic close between Mongolian and Chinese profiles of Beijing strains, but Mongolian isolates were grouped in specific cluster.

We determined that there were no significantly transmission of MDR strains from Russia to Mongolia. Our study confirms possibility of cross-border spread of Chinese strains in the past, but now Mongolia has own MDR strains source.

6.62 doi: 10.15789/2220-7619-2018-4-6.62

\section{MOLECULAR EPIDEMIOLOGY OF TUBERCULOSIS} IN EASTERN SIBERIA AND FAR EAST

S.N. Zhdanova ${ }^{1}$, M.K. Vinikurova ${ }^{2}$, A.A. Yakovlev $^{3}$, O.B. Ogarkov ${ }^{1,4}$

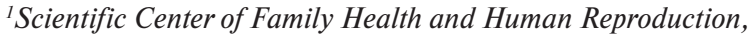
Irkutsk, Russia; ${ }^{2}$ Phthisiatry Research and Practice Center, Yakutsk, Russia: ${ }^{3}$ Vladivostok State Medical University, Vladivostok, Russia; ${ }^{4}$ Branch of the Educational Institution of Further Professional Education «Russian Medical Academy of Continuing Professional Education», Irkutsk, Russia

The highest burdens of tuberculosis in Russia have Asian regions. Beside of social and health care problems this area gained specific geographic spread of Mycobacterium tuberculosis (MBT) genotypes and high level of multi drug resistance (MDR). The aim was to carry out comparative evaluation of distribution epidemic MBT strains in Eastern Siberia and Far East of Russia.

We studied 1419 clinical MTB strains collected in Irkutsk region (598), Buryatia (306), Sakha (Yakutya) (351), Zabaykalsky Krai (65) and Primorsky Krai (99). RD 207, 105181 analysis and 24 loci MIRU-VNTR typing were applied for genotyping and the results were analyzed by MIRU-VNTRplus application. PCR-real time typing was used to identify subtype CC2/W148 by the specific deletion in the $k d p D$ gene.

Beijing strains were dominant and varied from $73.6 \%$ in the Irkutsk region (Primorsky Krai (72.7\%), Zabaykalsky Krai $(64.6 \%)$, Buryatia $(64.4 \%)$ ) to $43.3 \%$ in Sakha (Yakutia). Our study showed that minor Russian genotypes $\mathrm{T}(13.3 \%)$, Haarlem (7.4\%) and especially S (13.5\%) presented in Sakha (Yakutia) may reflect the MBT population spectrum existing in Russia before Beijing expansion in the $20^{\text {th }}$ century. Yakut's strains of genotype $\mathrm{S}$ is unique epidemic group with high level of MDR (77.4\% of all $\mathrm{S}$ isolates).

As there are in all Russian regions the subtypes $\mathrm{CC} 1$ and $\mathrm{CC} 2 / \mathrm{W} 148$ were prevailing among Beijing strains. We identified that fifty percent of the Beijing isolates belonged to $\mathrm{CC} 1$ group in Irkutsk region, Primorsky Krai and Sakha (Yakutia). Buryatia and Zabaykalsky Krai had unexpectedly low level of $\mathrm{CC} 1$, as there were endemic subtype Beijing BL7 (MIT 642) in a quarter of strains from the Beijing collection. The RD 181 deletion absence, the genetic variation of profiles and the presence common clusters of strains of different ethnic groups patients confirm the occurrence of stable circulation of endemic and epidemic BL7 (MIT 642) subtype among the domestic population. The size of subtype CC2/W148 cluster was various from $26.3 \%$ in Irkutsk region cohort [Zabaykalsky Krai (24.6\%), Primorsky Krai (22.2\%), Buryatia (14.0\%)] to $13.7 \%$ in Sakha (Yakutia). The significant frequency excess of primary MDR was detected only in CC2/W148 subtype vs other epidemic strains of Beijing and nonBeijing genotypes in all areas except Buryatia, where endemic subtype BL7 (MIT 642) shared MDR burden with CC2/ W148.

The genotypic structure of the MBT population in the Asian Russia mainly reflects the epidemic expansion features of the Beijing subtypes.

6.63 doi: 10.15789/2220-7619-2018-4-6.63

\section{MOLECULAR DIAGNOSTICS OF TUBERCULOSIS CLINICAL ASPECTS AND CHALLENGES OF IMPLEMENTATION}

\section{Zhuravlev}

St. Petersburg Research Institute of Phthisiopulmonology,

St. Petersburg, Russia

The modern standard of bacteriological diagnostics includes the following set of diagnostic procedures for simultaneous testing of a clinical specimen from a patient suspected of tuberculosis: luminescent microscopy, cultivation on solid media (Finn and Levenstein-Jensen), use of the liquid medium of the BAKTEK 320/960 analyzer, molecular genetic methods in various formats: real-time PCR, GenXpert TB-Rif, Hain test, biochips. The emergence of new technologies does not lead to the rejection of ineffective classical methods. So, in my opinion, the presence of molecular genetic methods in the algorithm of a clinical doctors allows to abandon the microscopy of a clinical material due to low sensitivity and specificity and thereby 
optimize costs. However, all the possibilities of molecular genetic methods are not taken into account. So, if the use of GenXpert TB-Rif is absolutely indicated in the case of acute progressive lesions with impaired vital functions in the patient, this technology is less effective with limited diagnostic processes without destruction and inferior to the effectiveness of real-time PCR. The spectrum of detected mutations associated with the development of drug resistance of the causative agent is not taken into account. If more sensitive and cost-effective PCR-based real-time test systems allow detecting only 8 mutations in three genes $(k a t G, \operatorname{inh} A, r p o B)$ that determine the drug resistance of the MBT in $90 \%$ of cases, the increase in migration will lead to an increase in cases of tuberculosis with other genetic markers of resistance. In clinical practice, the high risk of falsely positive results from molecular genetic studies in the analysis of material obtained from instrumental or surgical interventions is almost not considered. There is no discussion of the ineffectiveness of the use of molecular tests in the control of chemotherapy at early stages of treatment with a pronounced activity of the process. A clear algorithm is needed for each clinical task. 